\title{
Does complete renal denervation translate into superior clinical outcomes? Lessons learned from denervation of accessory renal arteries
}

\author{
Farrell O. Mendelsohn
}

Received: 20 December 2013/Accepted: 11 March 2014/Published online: 26 March 2014

(C) Springer-Verlag Berlin Heidelberg 2014

\begin{abstract}
Pre-clinical studies of renal denervation would suggest that the extent of renal nerve injury correlates with outcomes. The "completeness" of renal nerve injury following renal denervation correlates with treatment-based variables such as the depth of ablation, the number of ablations along the length of the artery, and the number of renal arteries successfully ablated. Renal denervation techniques targeting only main renal arteries may lead to suboptimal results in patients with accessory renal artery anatomy. Technological differences among the different systems may make some more suited for this common anatomical variant. The early clinical experience with renal denervation of accessory renal arteries highlights the importance of complete renal denervation for clinical success.
\end{abstract}

Keywords Renal denervation - Sympathetic nervous system · Hypertension · Renal artery · Accessory renal arteries

Renal denervation targets the sympathetic nerves located in the adventitia of the renal artery wall [1]. The nerves travel from the spinal cord along the artery and then to the kidney where extensive branching occurs. Pre-clinical studies would suggest that the degree of renal nerve injury correlates with outcomes (Mazor M. Efficacy of renal denervation is positively impacted by longitudinal treatments, TCT 2012, October 22-26, Miami, FL). Yet it is unlikely that complete denervation of the entire renal sympathetic nervous system is achieved with any renal artery ablation

F. O. Mendelsohn ( $\square)$

Interventional Hypertension Therapies Program, Cardiology PC, 801 Princeton Avenue, S.W., Suite 707, Birmingham, AL 35211, USA

e-mail: fmendelsohn@cardiologypc.com technology available. Variables such as the depth of ablation into the renal artery wall, the number of ablations along the length of the artery, and the number of renal arteries successfully ablated all correlate with the "completeness" of renal nerve injury and may affect clinical outcomes.

Hypertensive patients with accessory renal arteries who undergo renal denervation highlight the issue of whether complete interruption of the renal sympathetic nervous system correlates with changes in blood pressure. Most clinical trials of renal denervation have excluded patients with accessory renal arteries [2-6], yet these patients may constitute as much as $27 \%$ of the resistant hypertension population [7]. Id and colleagues recently reported their results of renal denervation using the Symplicity catheter system (Medtronic, Santa Rosa, CA) in patients both with and without accessory renal arteries [8]. The drop in officebased systolic blood pressure (SBP) at 6 months was significantly less in the patients with accessory renal arteries compared to those with only main renal artery trunks $(-6.2$ vs $-16.6 \mathrm{mmHg}, p=0.027$ ). The patients with accessory renal arteries were further subdivided into two groups: (1) those in whom all accessories underwent successful denervation and (2) those in whom only some or no accessory renal arteries could be denervated. The subgroup with complete accessory renal artery denervation experienced a slightly greater drop in SBP compared to patients with incomplete treatment of their accessory arteries $(-8.8$ vs $-4.1 \mathrm{mmHg}$ ).

Since the majority of renal nerves terminate at vascular structures within the kidney [9], nerve traffic to and from the kidney likely corresponds to the blood supply, traveling with both accessory and main renal arteries. So patients with accessory renal arteries may fail to respond if treated with a denervation technique targeting nerves only in the 


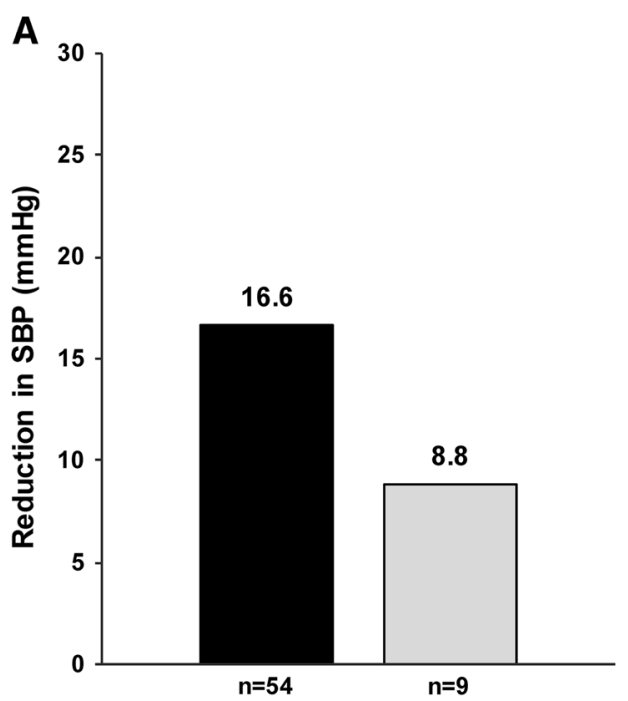

Fig. 1 Reduction in office-based systolic BP 6 months following renal denervation treatment-Black bars represent patients without accessory renal arteries who had renal denervation treatment. Gray bars represent patients with "complete" treatment of accessory renal

main renal artery-just as was observed by Id and colleagues [8]. Yet why, in their study, was the blood pressure-lowering response in the subgroup with "complete" denervation of accessory renal arteries less than that in the group with only main renal artery trunks (Fig. 1a)? This difference may in part be explained by limitations of the Symplicity renal denervation system for ablation of accessory renal arteries. The Symplicity system is composed of a single electrode monopolar radiofrequency ablation catheter connected to an automated generator. Energy passes from the generator through the catheter to an external grounding pad, which serves as a far field electrode. The generator delivers up to $8 \mathrm{~W}$ of power to the catheter tip causing heating of the adjacent tissue and consequent nerve injury. The system avoids overheating of the tissue by monitoring temperature from a thermistor near the electrode tip, shutting off power if the temperature exceeds a predetermined threshold. Cooling of the catheter tip from renal blood flow is critical to avoid overheating. In low-flow conditions with inadequate cooling, the generator will terminate energy delivery to prevent excessive tissue

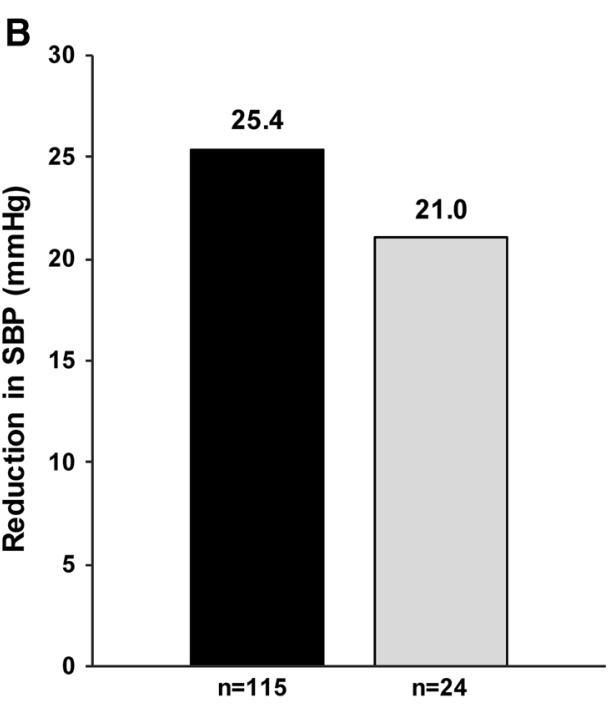

arteries (as defined by Id et al. [8]). a Reductions following treatment with the Symplicity system [8]. b Reductions following treatment with the Vessix System (Sievert H. REDUCE-HTN Clinical Study Interim 6 and 12 months data. TCT 2013. San Francisco, October 28, 2013)

damage. Ablation will be interrupted, and denervation efficiency potentially compromised.

Accessory renal arteries can present a low-flow condition with limited cooling from the bloodstream. Renal denervation systems such as Symplicity that require cooling may not be well-suited for the treatment of accessory renal arteries due to insufficient cooling from lower blood flow. By contrast, the Vessix Renal Denervation System (Boston Scientific Corporation, Natick, MA) does not require cooling from the bloodstream; its design integrates multiple elements that make cooling unnecessary. First, it delivers energy to the tissue via pairs of bipolar electrodes (Fig. 2) with efficient energy transfer leading to short treatment times. Second, the Vessix System controls temperature at $68{ }^{\circ} \mathrm{C}$ so that overheating cannot occur. Temperature control is enabled by a thermistor located between the positive and negative poles of each electrode pair (Fig. 2). And finally, the electrodes and thermistors are all mounted on a balloon catheter (Fig. 2). When inflated, the balloon apposes the electrodes and thermistors to the renal artery wall and simultaneously obstructs blood flow down

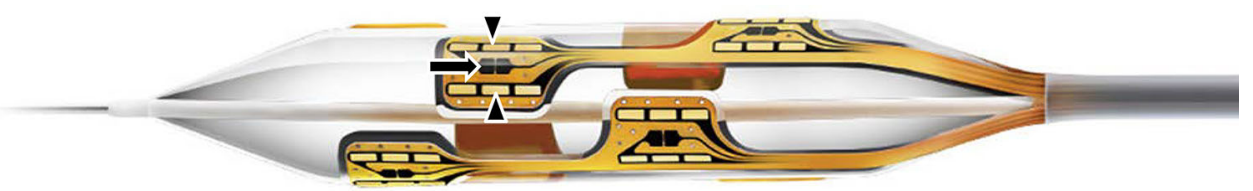

Fig. 2 Illustration of the helical array of bipolar electrodes (electrode pair indicated with arrowheads) and thermistors (arrow) mounted on the Vessix balloon catheter. Used with permission of Boston
Scientific Corporation $(2014$ Boston Scientific Corporation or its affiliates. All rights reserved 
the artery so that the thermistor readings provide a close approximation of tissue temperature without interference from the cooling effects of the bloodstream. All of these design elements combine into a denervation system that functions independent of blood flow conditions; and with catheter sizes suited for arteries as small as $3 \mathrm{~mm}$ in diameter, the Vessix device is optimized for renal denervation of accessory arteries.

The REDUCE-HTN post-market study tested the Vessix System in patients with resistant hypertension, including 24 patients with accessory renal arteries. In this subgroup where accessory renal arteries were treated, SBP dropped by $-21.0 \mathrm{mmHg}$ at 6 months (Sievert H. REDUCE-HTN Clinical Study Interim 6 and 12 months data, TCT 2013. San Francisco, October 28, 2013). In the absence of a direct head-to-head trial, these results cannot be fairly compared to those reported by Id and colleagues [8] (Fig. 1a, b), yet with one system the presence of accessory renal arteries appears to compromise efficacy while efficacy seems preserved with the other. The Vessix technology may result in more complete renal nerve injury despite variant renal artery anatomy.

The importance of denervation technology for complete renal nerve ablation cannot be overstated in light of the Symplicity HTN3 trial failure to meet its primary efficacy endpoint [10]. In the successful predicate studies to HTN3, namely HTN1 and HTN2, a catheter with a fixed distal curve (Arch) enabling renal artery wall apposition was replaced in HTN3 with a catheter (Flex) requiring operator manipulation for vessel wall contact. Less consistent catheter contact with the renal artery wall diminishes ablation effectiveness. The newer technology may have resulted in less renal nerve injury compromising the clinical trial results. It will be critical to understand whether these differences in renal denervation technologies lead to different degrees of nerve injury and translate into variations in clinical outcomes. Complete renal denervation should be the goal.
Acknowledgments I thank Margaret Taber and Elizabeth Davis (Boston Scientific Corporation, Marlborough, MA) for assistance with figure preparation and with preparing the manuscript for submission.

Disclosure Consulting fees have been received from Medtronic and Boston Scientific/Vessix. No funding was received for preparing this work; open access was funded by Boston Scientific Corporation.

\section{References}

1. Atherton DS, Deep NL, Mendelsohn FO (2012) Micro-anatomy of the renal sympathetic nervous system: a human postmortem histologic study. Clin Anat 25:628-633

2. Krum H, Schlaich M, Whitbourn R (2009) Catheter-based renal sympathetic denervation for resistant hypertension: a multicentre safety and proof-of-principle cohort study. Lancet 373:12751281

3. Symplicity HTN-2 Investigators (2010) Renal sympathetic denervation in patients with treatment resistant hypertension (the Symplicity HTN-2 Trial): a randomized controlled trial. Lancet 376:1903-1909

4. Kandzari DE, Bhatt DL, Sobotka PA et al (2012) Catheter-based renal denervation for resistant hypertension: rationale and design of the SYMPLICITY HTN-3 Trial. Clin Cardiol 35:528-535

5. Vogel B, Kirchberger M, Zeier M et al (2014) Renal sympathetic denervation therapy in the real world: results from the Heidelberg registry. Clin Res Cardiol 103:117-124

6. Mahfoud F, Luscher TF, Andersson B et al (2013) Expert consensus document from the European Society of Cardiology on catheter-based renal denervation. Eur Heart J 34:2149-2157

7. Satyapal KS, Haffejee AA, Singh B, Ramsaroop L, Robbs JV, Kalideen JM (2001) Additional renal arteries: incidence and morphometry. Surg Radiol Anat 23:33-38

8. Id D, Kaltenbach B, Bertog SC et al (2013) Does the presence of accessory renal arteries affect the efficacy of renal denervation? J Am Coll Cardiovasc Interv 6:1085-1091

9. Barajas L, Powers K (1990) Monoaminergic innervation of the rat kidney: a quantitative study. Am J Physiol 259:F503-F511

10. Press release (2014) Medtronic announces U.S. renal denervation pivotal trial fails to meet primary efficacy endpoint while meeting primary safety endpoint. http://newsroom.medtronic.com/phoe nix.zhtml?c=251324\&p=irol-newsArticle $\&$ id $=1889335$. Accessed 04 March 2014 